\title{
Using an ANN Approach to Estimate Output Power and PAE of A Modified Class-F Power Amplifier
}

\author{
Mohammad (Behdad) Jamshidi \\ Department of Electromechanical Engineering and \\ Power Electronics (KEV), \\ University of West Bohemia \\ Pilsen, the Czech Republic \\ jamshidi@kev.zcu.cz \\ Jakub Talla \\ Department of Electromechanical Engineering and \\ Power Electronics (KEV), \\ University of West Bohemia \\ Pilsen, the Czech Republic \\ talic@rice.zcu.cz
}

\author{
Saeed Roshani \\ Department of Electrical Engineering, Kermanshah \\ Branch, Islamic Azad University, Kermanshah, Iran \\ s_roshany@yahoo.com
}

Sobhan Roshani*

Department of Electrical Engineering, Kermanshah Branch, Islamic Azad University, Kermanshah, Iran sobhan_roshany@yahoo.ca

*Corresponding Author

\begin{abstract}
In this paper, an efficient Class-F power amplifier (PA) is designed, simulated and modeled. This type of amplifier has nonlinear behaviors and uses tuning and controlling harmonics as the most important mechanism to increase efficiency. Feedforward artificial neural network (ANN) model is proposed to predict and estimate the nonlinear output of the power amplifier. The designed amplifier operates at $900 \mathrm{MHz}$, with $18 \mathrm{~dB}$ gain and $70 \%$ Power-Added Efficiency (PAE). In the design process, the artificial neural network model is used to predict PAE and output power parameters as a function of input power, drain voltage and gate voltage of the applied transistor (DC Biasing voltages). The obtained mean relative errors (MREs) are less than $0.03 \%$ and $0.09 \%$ for the predicted output power and PAE parameters.
\end{abstract}

Keywords-component; Artificial neural network; Class-F power amplifier; Power added efficiency (PAE)

\section{INTRODUCTION}

PAs are high power-consuming elements in communications. Therefore, efficiency is an important factor in amplifier design [1-4]. Switching power amplifiers are good choices for high-efficiency applications. Also, Class-F is a good choice of switching amplifier, because of its high efficiency. Class-F amplifier is the harmonic controlled type of amplifiers [5-7]. In another word, the switch voltage and current are formed using different harmonics in Class-F amplifier. The switch voltage is squared waveform, while the switch current is a half-sinusoidal waveform in the Class-F amplifier.

Usually analyzing of switching amplifiers leads to complex equations. In most cases, there is not any closed-form solution and the equations should be solved numerically. Therefore, designing of the switching amplifiers is a time-consuming procedure, especially when the parasitic transistor elements are considered[2].

The Class-F amplifier is a circuit with output harmonic control circuit. This harmonic control circuit adjusts the harmonics in the transistor voltage and current waveforms to shape the transistor waveforms. The transistor squared voltage and half-sinusoidal current waveforms are shaped in the such way that they are not overlapped in the time domain, therefore, the efficiency of the amplifier will be increased. This advantage causes Class-F amplifier to be desirable choice in the high efficiency applications [8-10].

Artificial intelligence is an excellent solution to ease the design procedure and modeling in several engineering problems [11-15]. Also, ANNs have been used to model the power amplifiers [16-19]. There are several conventional ANN networks which are used in electronics filed, such as MLP (multilayer perceptron) [16] and spiking neural networks [20].

\section{AMPLIFIER DESIGN}

The block diagram of the Class-F PA is shown in Fig. 1(a). As seen, the PA has two matching networks, which transfer input and output impedances (50 ohms) to the desired values at the gate and drain of the applied transistor. These desired values obtained from source-pull and load-pull analysis. The output matching networks, not only transfer output impedance to the desired value but also control the harmonics in the circuit. The harmonics control circuit (HCC) is the important part in the Class-F PA. HCC passes even voltage harmonics and suppresses odd harmonics, so the voltage and current waveforms will be shaped as shown in Fig. 1(b). The drain voltage and drain current of a typical Class-F amplifier can be written as follows [21]: 


$$
\begin{aligned}
& V_{D}(\theta)=V_{D D}+V_{m} \sin (\theta)+V_{3 m} \sin (3 \theta)+V_{5 m} \sin (5 \theta) \ldots \\
& i_{D}(\theta)=I_{D}-I_{m} \sin (\theta)-I_{2 m} \sin (2 \theta)-I_{4 m} \sin (4 \theta) \ldots
\end{aligned}
$$

In equations (1) and (2), $V_{\mathrm{D}}$ and $I_{\mathrm{D}}$ stand for $\mathrm{DC}$ drain voltage and current, respectively. Also, $V_{\mathrm{im}}$ and $i_{\text {im }}$ are the $i_{\text {th }}$ harmonics drain voltage and current amplitude, respectively. Moreover, $\theta=\omega t$ and $\omega$ is the fundamental angular frequency.
The proposed amplifier is shown in Fig. 2. In the designed amplifier microstrip transmission lines (TLs), pHEMT transistor and capacitors are used. Transmission Lines TL1-TL3 is used as an input matching network and TL8-TL10 are used as output matching network, which works as harmonics control circuit (HCC) simultaneously. Transmission Lines TL4-TL5 are used as DC drain bias line. In this design $5 \mathrm{~V}, \mathrm{DC}$ voltage is used for drain and $-0.75 \mathrm{~V}$, DC voltage is used for gate voltage. Transmission Lines TL6-TL7 are used as DC gate bias line.

Figure 1. (a) Block diagram and (b) drain waveforms of typical Class-F PA

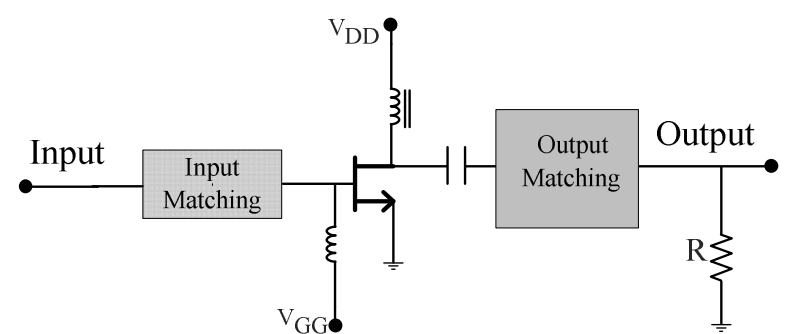

(a)

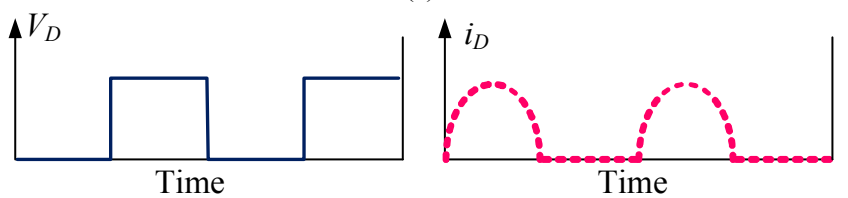

(b)

Figure 2. The schematic structure of the designed Class-F amplifier.

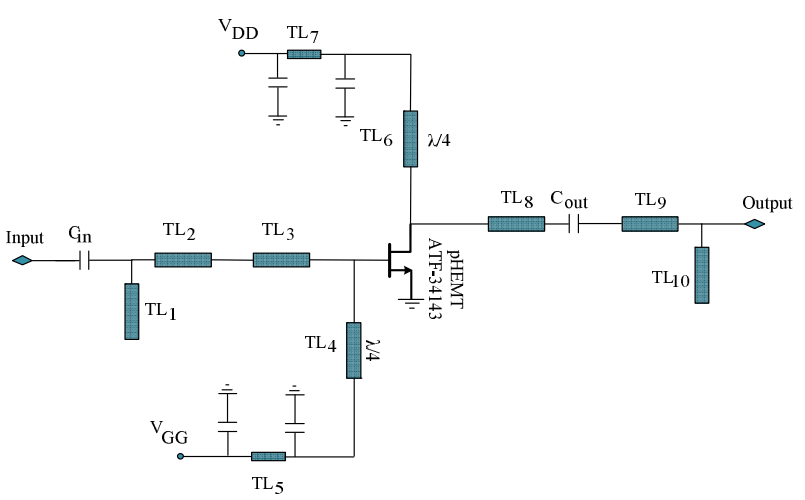

TABLE I. DESCRIPTION OF THE APPLIED PARAMETERS

\begin{tabular}{|c|l|}
\hline Parameter & \multicolumn{1}{c|}{ Discription } \\
\hline $\mathrm{V}_{\mathrm{DD}}$ & DC voltage for the drain of the amplifier \\
\hline $\mathrm{V}_{\mathrm{GG}}$ & DC gate voltage for the gate of amplifier \\
\hline$V_{\mathrm{D}}$ & Drain voltage of the transistor \\
\hline$i_{\mathrm{D}}$ & Drain Current of the transistor \\
\hline$V_{\mathrm{im}}$ & Magnitude of the $\mathrm{i}^{\text {th }}$ voltage harmonic \\
\hline$V_{\mathrm{im}}$ & Magnitude of the $\mathrm{i}^{\text {th }}$ current harmonic \\
\hline $\mathrm{C}_{\mathrm{in}}$ & Capacitance at the input of the amplifier \\
\hline $\mathrm{C}_{\text {out }}$ & Capacitance at the output of the amplifier \\
\hline $\mathrm{Tl}_{\mathrm{i}}$ & Index for the $\mathrm{i}^{\text {th }}$ transmission line \\
\hline
\end{tabular}

Description of the applied parameters in the paper is listed in Table I. According to the circuit of the proposed amplifier, the LC equivalent circuit (LCEC) of the amplifier is extracted, which is shown in Fig. 3. In the presented LCEC, the inductors and capacitors are named corresponding to the transmission lines TL1-TL10. The amplifier layout, based on the circuit schematic diagram, is shown in Fig. 4. The scattering parameter (S21) of the designed Class-F amplifier is shown in Fig. 5. The scattering parameters for a device describe the electrical behaviour of a linear device, which can be defined by a matrix. For a two port amplifier scattering parameters can be written in a $2 \times 2$ matrix. In the small signal condition, the amplifier can be assumed as a linear device, so the S21 parameter shows the small signal gain of the amplifier. The achieved small signal gain shows good performance of the proposed amplifier at $900 \mathrm{MHz}$ frequency. The value of small-signal gain, at the main frequency, is about $18 \mathrm{~dB}$. The gain and PAE parameters are shown in Fig. 6. As seen, the large signal gain of the proposed 
PA is about $17.7 \mathrm{~dB}$, which confirmed the small-signal gain value. The PAE of the designed amplifier is about $70 \%$ for $15 \mathrm{dBm}$ input power at $900 \mathrm{MHz}$ frequency. The amplifier output power at main frequency is depicted in Fig. 7. As seen in this figure, the 1-dB point of the designed PA is about $30 \mathrm{dBm}$, which shows an acceptable linear operating range. Fig. 8 shows the drain waveforms of the amplifier. As seen the drain voltage and current are approximately square and half-sinusoidal waveforms.

\section{NEURAL NeTWORK MODELING}

As mentioned, the artificial neural network is the proper technique to model electrical devices such as microwave devices and power amplifiers. The parameters which are considered for the input of the neural network are 1- input power Pin $(\mathrm{dBm}), 2-\mathrm{DC}$ gate bias voltage $\mathrm{Vg}(\mathrm{V})$ and $\mathrm{DC}$ drain bias $\mathrm{Vd}(\mathrm{V})$. Also, the parameters which are considered for output of the neural network are 1- output power Pout $(\mathrm{dBm})$ and 2- power-added efficiency (\%). The considered neural network for modeling of the amplifier is shown in Fig. 9.

The multi-layer perceptron (MLP) neural network is considered in this paper to model the power amplifier. The presented MLP in this paper consists of two hidden layers with five and six neurons in the first and second hidden layers, respectively. Fig. 10 shows the architecture of the proposed MLP network. The proposed ANN ran more than 200 times and then the corresponding values of MRE for test data are reported in Table II.

Figure 3. The LCEC of the designed Class-F amplifier.

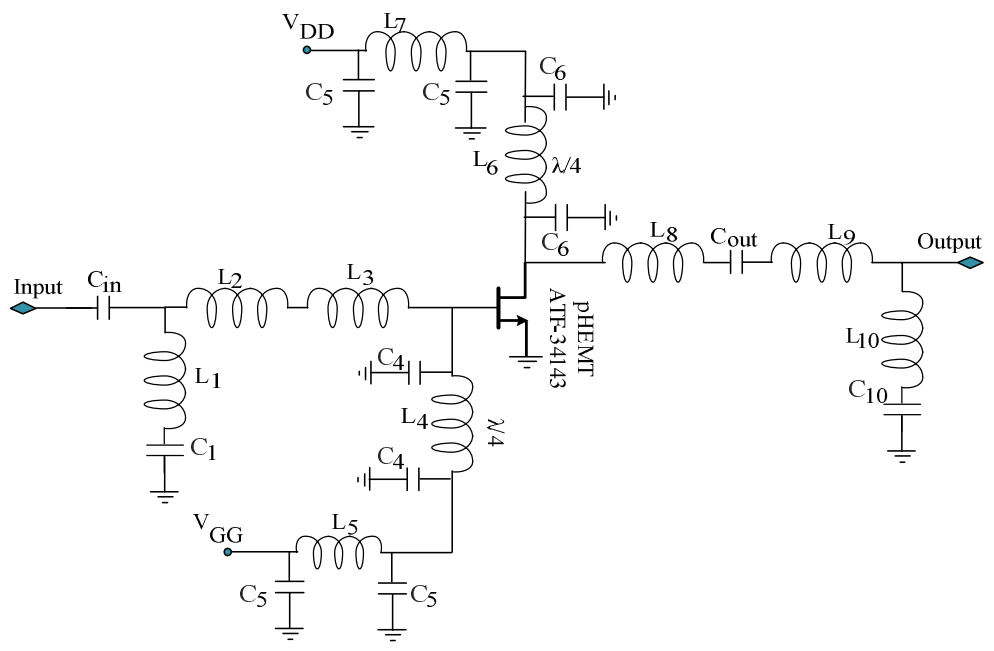

Figure 4. The layout of the designed amplifier

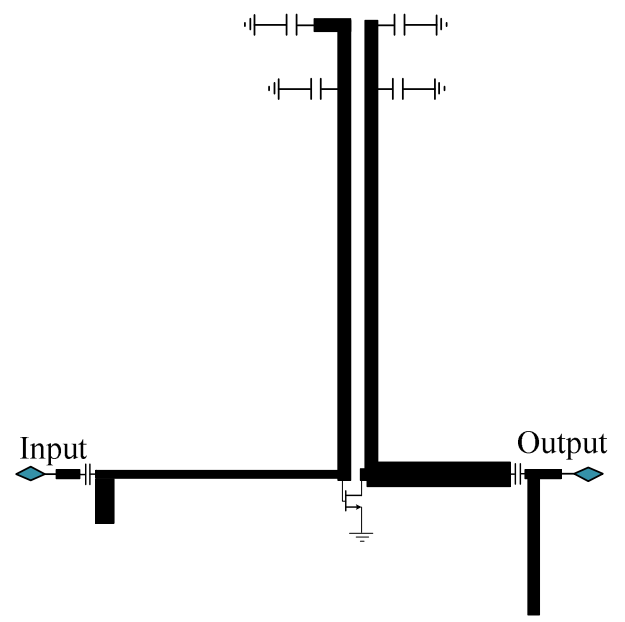

Figure 5. S21-parameter of the designed Class-F amplifier.

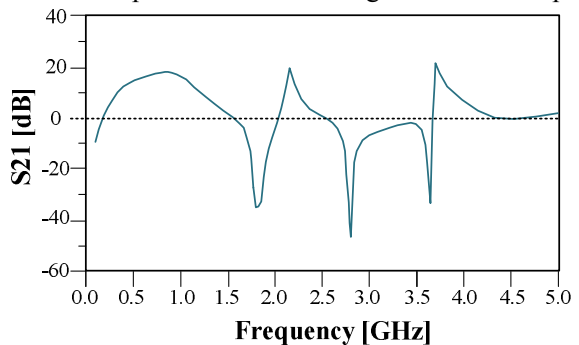

Figure 6. The gain and PAE parameters of the designed Class-F amplifier at $0.9 \mathrm{GHz}$.

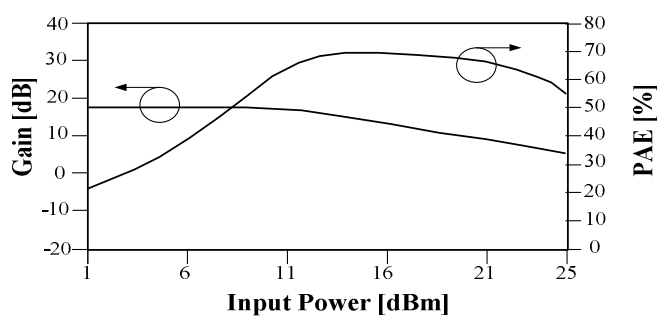




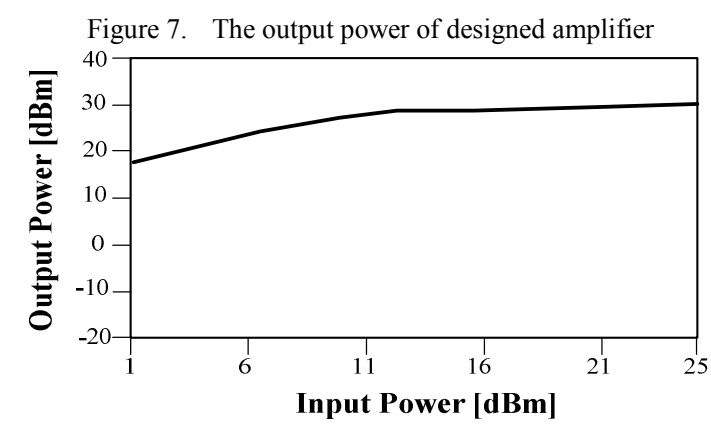

Figure 8. The drain waveforms of the amplifier.

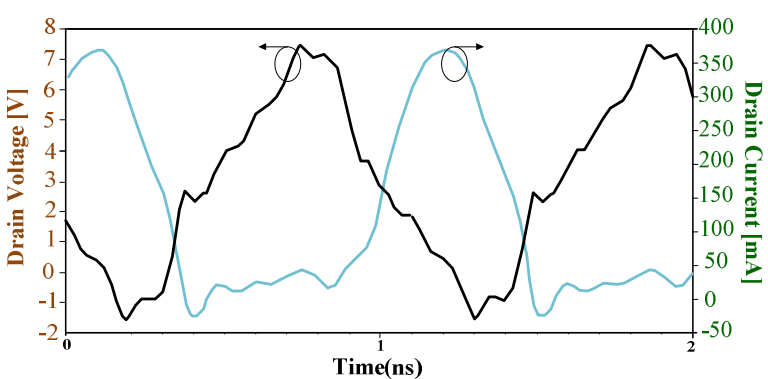

The mean relative error (MRE) percentage is written as follows.

$$
\operatorname{MRE}=\frac{\sum_{i=1}^{N}\left|Y_{\mathrm{Re} i}-Y_{\mathrm{Pr} i}\right| /\left|Y_{\mathrm{Re} i}\right|}{N} \times 100
$$

where, in this equation " $\mathrm{Y}$ " represents the data value and "N" is the total data number. Also, the "Re" and "Pr" indices represent the real and predicted values. 171 data are considered for train and 57 data are considered for the test process of the modelling.

Figure 9. Considered neural network for modelling of the amplifier

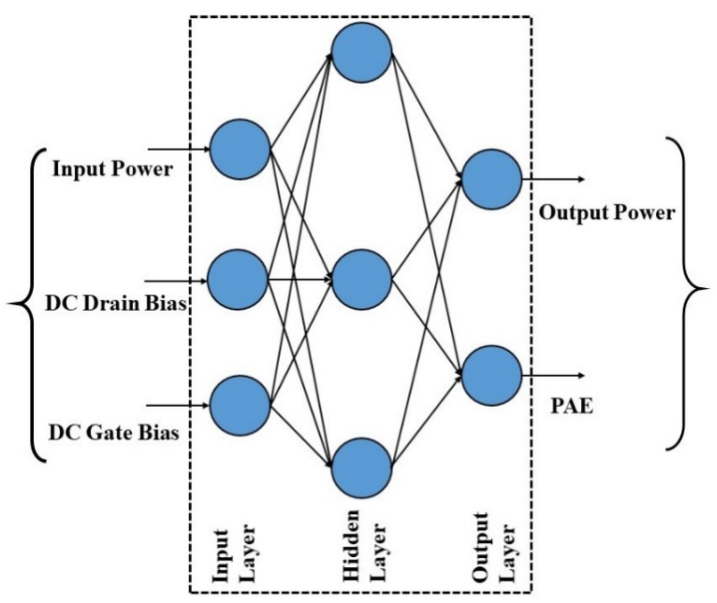

Figure 10. Arcitecture of the proposed MLP neural network model

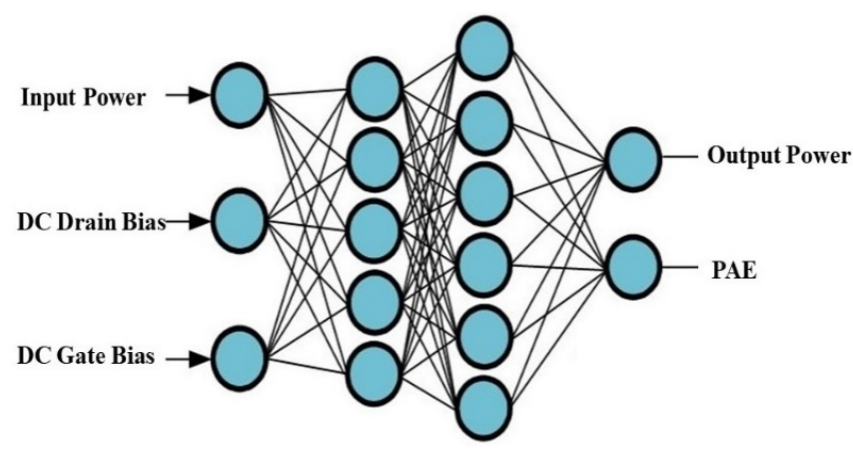

The regression diagrams for the predicted and real values of the output power in the proposed model are depicted in Fig. 11. The Real and predicted output power in the presented model is depicted in Fig. 12.

TABLE II. THE RESULTS OF THE PROPOSED MODEL FOR TRAIN AND TEST PROCEDURES

\begin{tabular}{|c|c|c|}
\hline Error-values & P $_{\text {out }}(\mathbf{d B m})$ & PAE (\%) \\
\hline MRE Train \% & 0.03 & 0.09 \\
\hline MRE Test \% & 0.04 & 0.23 \\
\hline
\end{tabular}

Figure 11. Regression diagrams for the predicted and real values of the output power in the proposed model: (a) Train and (b) Test steps.

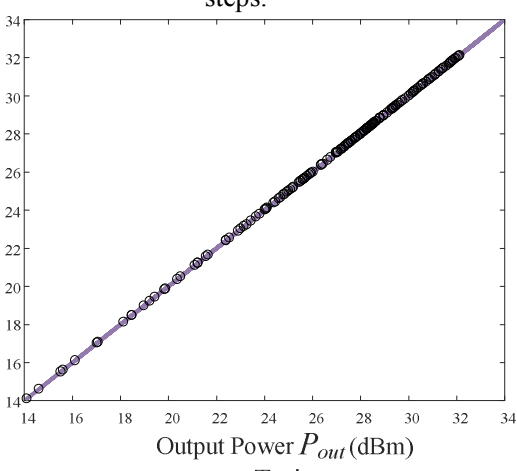

Train

(a)

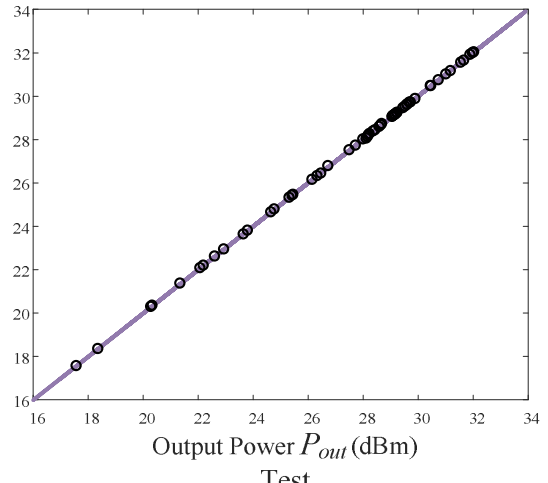

(b)

Real and predicted power added efficiency and output power for drain voltage of $5 \mathrm{~V}$ and gate voltage of $-0.75 \mathrm{~V}$ is illustrated in Fig. 12. As can be seen in this Figure, the ANN has predicted the power added efficiency and output power precisely. 
Figure 12. Real and predicted power added efficiency in the proposed model: (a) Train and (b) Test steps.

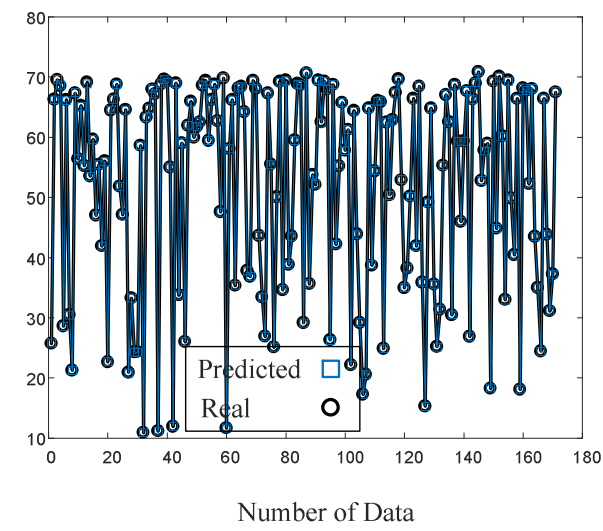

(a)

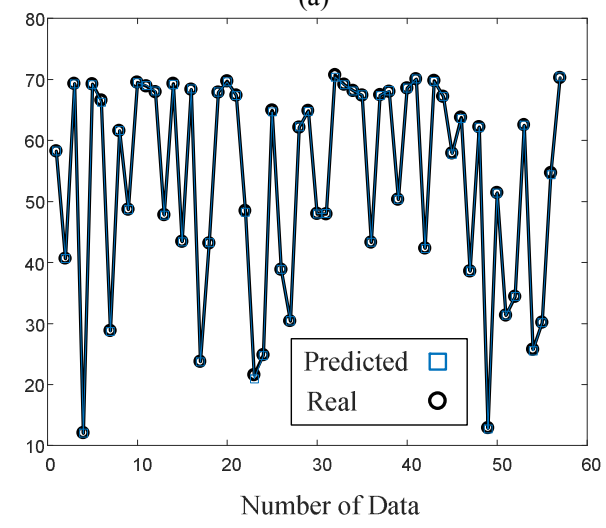

(b)

Figure 13. Real and predicted power added efficiency and output power

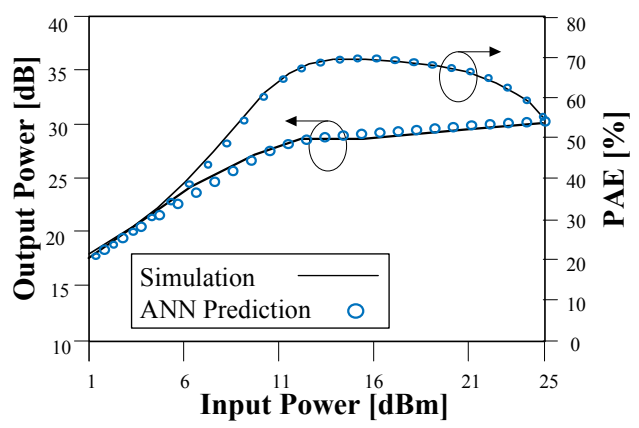

IV. CONCLUSION

A Class-F PA is designed and modeled using neural networks. The proposed neural network model can predict the amplifier parameter correctly. The predicated parameters of the amplifier are close to the real values. Finally, the mean relative errors of the predicted parameters are calculated which are less than $0.03 \%$ and $0.09 \%$ for the predicted output power and PAE parameters.

\section{ACKNOWLEDGMENT}

This research has been supported by the Ministry of Education, Youth and Sports of the Czech Republic under the project OP VVV Electrical Engineering Technologies with High-Level of Embedded Intelligence CZ.02.1.01/0.0/0.0/18_069/0009855.

\section{REFERENCES}

[1] A. Pirasteh, S. Roshani, and , S. Roshani, "A modified ClassF power amplifier with miniaturized harmonic control circuit," AEU-International Journal of Electronics and Communications, 2018, 97, pp. 202-209

[2] M. Hayati, S. Roshani, S. Roshani, M.K. Kazimierczuk, and H. Sekiya, "Design of class E power amplifier with new structure and flat top switch voltage waveform," IEEE Transactions on Power Electronics, 2017, 33, (3), pp. 25712579

[3] S. Roshani, M. Hayati, S. Setayeshi, S. Roshani, and G. Mohamadpour, "A miniaturized harmonic suppressed power amplifier integrated with lowpass filter for long term evolution application," Analog Integrated Circuits and Signal Processing, 2016, 89, (1), pp. 197-204

[4] S. Roshani, and S. Roshani, "Design of a high efficiency Class-F power amplifier with large signal and small signal measurements," Measurement, 2020, 149, pp. 106991

[5] S.Y. Zheng, Z.W. Liu, X.Y. Zhang, X.Y. Zhou, and W.S. Chan, "Design of ultrawideband high-efficiency extended continuous Class-F power amplifier," IEEE Transactions on Industrial Electronics, 2017, 65, (6), pp. 4661-4669

[6] T. Sharma, R. Darraji, F. Ghannouchi, and N. Dawar, "Generalized continuous Class-F harmonic tuned power amplifiers," IEEE Microwave and Wireless Components Letters, 2016, 26, (3), pp. 213-215

[7] J. Kim, "Highly efficient asymmetric Class-F- 1/F GaN Doherty amplifier," IEEE Transactions on Microwave Theory and Techniques, 2018, 66, (9), pp. 4070-4077

[8] M. Hookari, S. Roshani, and S. Roshani, "High efficiency balanced power amplifier using miniaturized harmonics suppressed coupler," International Journal of RF and Microwave Computer Aided Engineering, 2020, In Press, doi: doi.org/10.1002/mmce. 22252

[9] A. Pirasteh, S. Roshani, S. Roshani, "Compact microstrip lowpass filter with ultrasharp response using a square-loaded modified T-shaped resonator," Turkish Journal of Electrical Engineering \& Computer Sciences, Jul 2018, 26, (4), pp. 1736-46.

[10] A.R. Zirak, and S. Roshani, "A Reduced Switch Voltage Stress Class E Power Amplifier Using Harmonic Control Networks," International Journal of Advanced Computer Science and Applications, 2016, (7), pp. 38-42

[11] M.B. Jamshidi, A. Lalbakhsh, S. Lotfi, H. Siahkamari, B. Mohamadzade, and J. Jalilian, "A neuro based approach to designing a Wilkinson power divider", International Journal of RF and Microwave Computer Aided Engineering, 2020, 30, (3), pp. e22091

[12] M.B. Jamshidi, A. Lalbakhsh, B. Mohamadzade, H. Siahkamari, and S.M.H. Mousavi, "A novel neural-based approach for design of microstrip filters," AEU-International Journal of Electronics and Communications, 2019, 110, pp. 152847.

[13] M.B. Jamshidi, and S. Rostami, "A dynamic artificial neural network approach to estimate thermal behaviors of li-ion batteries," Automatic Control and Intelligent Systems (I2CACIS), 2017 IEEE 2nd International Conference on (IEEE, 2017, edn.), pp. 116-121

[14] M.B. Jamshidi, A. Lalbakhsh, et al. "Artificial Intelligence and COVID-19: Deep Learning Approaches for Diagnosis and Treatment. IEEE Access, June 2020. 99, 1-1 DOI: 10.1109/ACCESS.2020.3001973

[15] A. Lalbakhsh, M.B. Jamshidi, et al. "A Compact Lowpass Filter for Satellite Communication Systems Based on Transfer Function Analysis" AEÜ - International Journal of Electronics and Communications, 2020.

[16] S. Roshani, and S. Roshani, "Two-Section Impedance Transformer Design and Modeling for Power Amplifier Applications," Applied Computational Electromagnetics Society Journal, 2017, 32, (11), pp. 1042-1047

[17] A.R. Zirak, and S. Roshani, "Design and Modeling of RF Power Amplifiers with Radial Basis Function Artificial Neural Networks," International Journal of Advanced Computer Science and Applications, 2016, 7, (6), pp. 227-231 
[18] R. Hongyo, Y. Egashira, T.M. Hone, and K. Yamaguchi, "Deep neural network-based digital predistorter for Doherty power amplifiers," IEEE Microwave and Wireless Components Letters, 2019, 29, (2), pp. 146-148

[19] Z. Liu, X. Hu, T. Liu, X. Li, W. Wang, and F.M. Ghannouchi, "Attention-Based Deep Neural Network Behavioral Model for Wideband Wireless Power Amplifiers," IEEE Microwave and Wireless Components Letters, 2019, 39, (1), pp. 82-85

[20] J. Li, H. Xu, S.Y. Sun, S. Liu, N. Li, Q. Li, H. Liu, and Z. Li, "Enhanced Spiking Neural Network with forgetting phenomenon based on electronic synaptic devices,"
Neurocomputing,

2019.

DOI::

doi.org/10.1016/j.neucom.2019.09.030

[21] FH. Raab, "Class-F power amplifiers with maximally flat waveforms," IEEE Transactions on Microwave Theory and Techniques. 1997 Nov;45(11):2007-12. 